\title{
Ferromagnetic and antiferromagnetic order in bacterial vortex lattices
}

\author{
Hugo Wioland ${ }^{1,2 \dagger}$, Francis G. Woodhouse ${ }^{1,3 \dagger}$, Jörn Dunkel ${ }^{4}$ and Raymond E. Goldstein ${ }^{1 \star}$
}

\begin{abstract}
Despite their inherently non-equilibrium nature', living systems can self-organize in highly ordered collective states ${ }^{2,3}$ that share striking similarities with the thermodynamic equilibrium phases ${ }^{4,5}$ of conventional condensed-matter and fluid systems. Examples range from the liquid-crystal-like arrangements of bacterial colonies ${ }^{6,7}$, microbial suspensions $s^{8,9}$ and tissues ${ }^{10}$ to the coherent macro-scale dynamics in schools of fish ${ }^{11}$ and flocks of birds ${ }^{12}$. Yet, the generic mathematical principles that govern the emergence of structure in such artificial $^{13}$ and biological ${ }^{6-9,14}$ systems are elusive. It is not clear when, or even whether, well-established theoretical concepts describing universal thermostatistics of equilibrium systems can capture and classify ordered states of living matter. Here, we connect these two previously disparate regimes: through microfluidic experiments and mathematical modelling, we demonstrate that lattices of hydrodynamically coupled bacterial vortices can spontaneously organize into distinct patterns characterized by ferro- and antiferromagnetic order. The coupling between adjacent vortices can be controlled by tuning the inter-cavity gap widths. The emergence of opposing order regimes is tightly linked to the existence of geometry-induced edge currents ${ }^{15,16}$, reminiscent of those in quantum systems ${ }^{17-19}$. Our experimental observations can be rationalized in terms of a generic lattice field theory, suggesting that bacterial spin networks belong to the same universality class as a wide range of equilibrium systems.
\end{abstract}

Lattice field theories (LFTs) have been instrumental in uncovering a wide range of fundamental physical phenomena, from quark confinement in atomic nuclei ${ }^{20}$ and neutron stars ${ }^{21}$ to topologically protected states of matter ${ }^{22}$ and transport in novel magnetic ${ }^{23}$ and electronic $c^{24,25}$ materials. LFTs can be constructed either by discretizing the spacetime continuum underlying classical and quantum field theories ${ }^{20}$, or by approximating discrete physical quantities, such as the electron spins in a crystal lattice, through continuous variables. In equilibrium thermodynamics, LFT approaches have proved invaluable both computationally and analytically, for a single LFT often represents a broad class of microscopically distinct physical systems that exhibit the same universal scaling behaviours in the vicinity of a phase transition $^{4,26}$. However, until now there has been little evidence as to whether the emergence of order in living matter can be understood within this universality framework. Our combined experimental and theoretical analysis reveals a number of striking analogies between the collective cell dynamics in bacterial fluids and known phases of condensed-matter systems, thereby implying that universality concepts may be more broadly applicable than previously thought.

To realize a microbial non-equilibrium LFT, we injected dense suspensions of the rod-like swimming bacterium Bacillus subtilis into shallow polydimethyl siloxane (PDMS) chambers in which identical circular cavities are connected to form one- and twodimensional (2D) lattice networks (Fig. 1, Supplementary Fig. 6 and Methods). Each cavity is $50 \mu \mathrm{m}$ in diameter and $18 \mu \mathrm{m}$ deep, a geometry known to induce a stably circulating vortex when a dense bacterial suspension is confined within an isolated flattened droplet $^{15}$. For each cavity $i$, we define the continuous vortex spin variable $V_{i}(t)$ at time $t$ as the total angular momentum of the local bacterial flow within this cavity, determined by particle imaging velocimetry (PIV) analysis (Fig. 1b,f; Supplementary Movies 1 and 2 and Methods). To account for the effect of oxygenation variability on suspension motility ${ }^{9}$, flow velocities are normalized by the overall root-mean-square (r.m.s.) speed measured in the corresponding experiment. Bacterial vortices in neighbouring cavities interact through a gap of predetermined width $w$ (Fig. 1f). To explore different interaction strengths, we performed experiments over a range of gap parameters $w$ (Methods). For square lattices, we varied $w$ from 4 to $25 \mu \mathrm{m}$ and found that for all but the largest gaps, $w \leq w_{*} \approx 20 \mu \mathrm{m}$, the suspensions generally self-organize into coherent vortex lattices, exhibiting domains of correlated spins whose characteristics depend on coupling strength (Fig. 1a,e). If the gap size exceeds $w_{*}$, bacteria can move freely between cavities and individual vortices cease to exist. Here, we focus exclusively on the vortex regime $w<w_{*}$ and quantify preferred magnetic order through the normalized mean spin-spin correlation $\chi=\left\langle\Sigma_{i \sim j} V_{i}(t) V_{j}(t) / \Sigma_{i \sim j}\left|V_{i}(t) V_{j}(t)\right|\right\rangle$, where $\Sigma_{i \sim j}$ denotes a sum over pairs $\{i, j\}$ of adjacent cavities and $\langle\cdot\rangle$ denotes time average.

Square lattices reveal two distinct states of preferred magnetic order (Fig. 1a,e,i), one with $\chi<0$ and the other with $\chi>0$, transitioning between them at a critical gap width $w_{\text {crit }} \approx 8 \mu \mathrm{m}$ (Fig. 1j). For subcritical values $w<w_{\text {crit }}$, we observe an antiferromagnetic phase with anti-correlated $(\chi<0)$ spin orientations between neighbouring chambers on average (Fig. 1a and Supplementary Movie 1). By contrast, for $w>w_{\text {crit }}$, spins are positively correlated $(\chi>0)$ in a ferromagnetic phase (Fig. 1e and Supplementary Movie 2). Noting that the r.m.s. spin $\left\langle V_{i}(t)^{2}\right\rangle^{1 / 2}$ decays only slowly with increasing gap width $w \rightarrow w_{*}$ (Fig. 1k), and that the chambers do not impose any preferred handedness

${ }^{1}$ Department of Applied Mathematics and Theoretical Physics, University of Cambridge, Wilberforce Road, Cambridge CB3 OWB, UK. ${ }^{2}$ Institut Jacques Monod, Centre Nationale pour la Recherche Scientifique (CNRS), UMR 7592, Université Paris Diderot, Sorbonne Paris Cité, F-75205 Paris, France. ${ }^{3}$ Faculty of Engineering, Computing and Mathematics, The University of Western Australia, 35 Stirling Highway, Crawley, Perth, Western Australia 6009, Australia. ${ }^{4}$ Department of Mathematics, Massachusetts Institute of Technology, 77 Massachusetts Avenue, Cambridge, Massachusetts 02139-4307, USA. ${ }^{\dagger}$ Present addresses: Institut Jacques Monod, Centre Nationale pour la Recherche Scientifique (CNRS), UMR 7592, Université Paris Diderot, Sorbonne Paris Cité, F-75205 Paris, France (H.W.); Department of Applied Mathematics and Theoretical Physics, University of Cambridge, Wilberforce Road, 


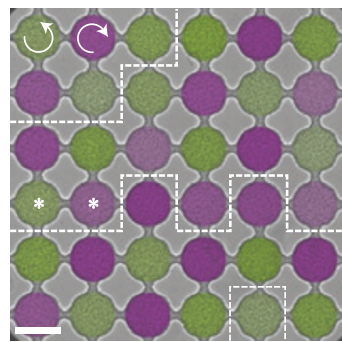

e

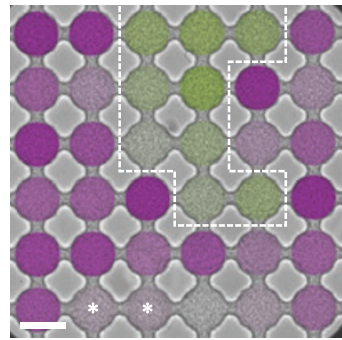

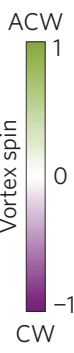

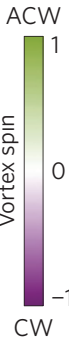

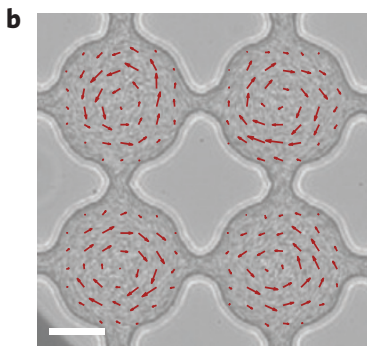

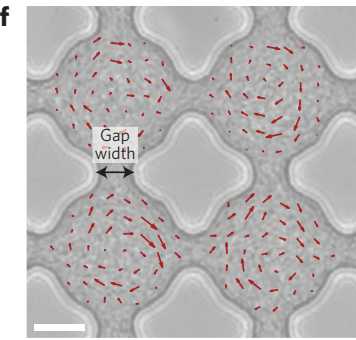

c

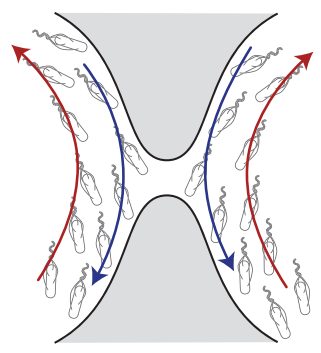

g

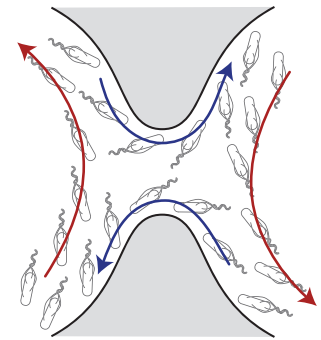

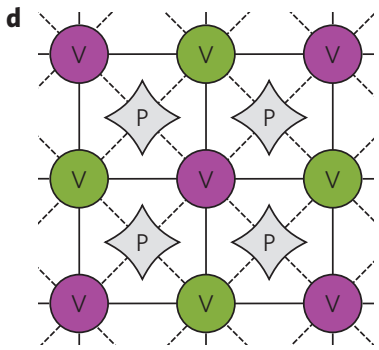

h

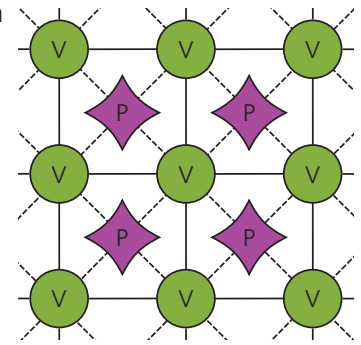

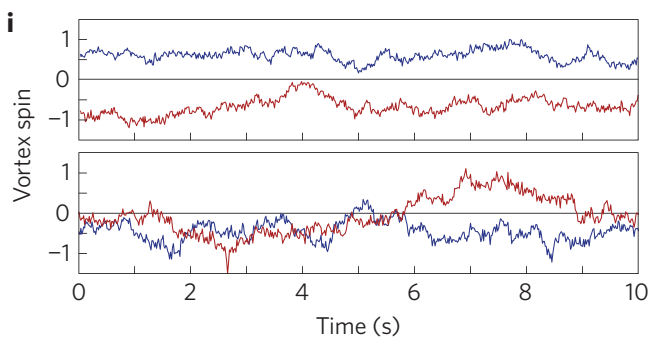
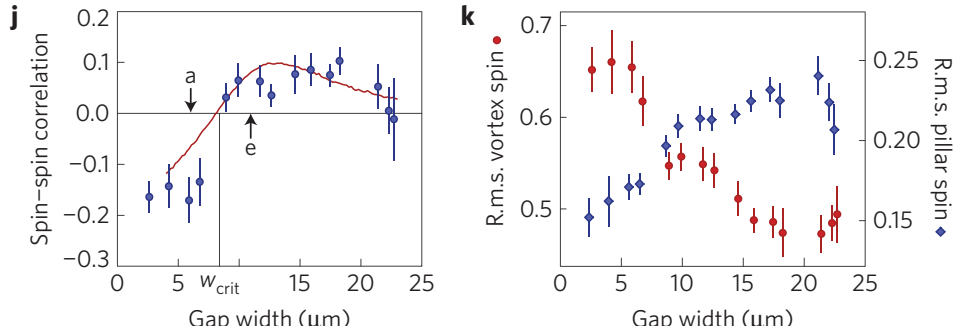

Figure 1 | Edge currents determine antiferromagnetic and ferromagnetic order in a square lattice of bacterial vortices. a, Three domains of antiferromagnetic order highlighted by dashed white lines (gap width $w=6 \mu \mathrm{m}$ ). Scale bar, $50 \mu \mathrm{m}$. Overlaid false colour shows spin magnitude (see Supplementary Movie 1 for raw data). b, Bacterial flow PIV field within an antiferromagnetic domain (Supplementary Movie 1). For clarity, not all velocity vectors are shown. Largest arrows correspond to speed $40 \mu \mathrm{m} \mathrm{s}{ }^{-1}$. Scale bar, $20 \mu \mathrm{m}$. c, Schematic of bacterial flow circulation in the vicinity of a gap. For small gaps $w<w_{\text {crit }}$, bacteria forming the edge currents (blue arrows) swim across the gap, remaining in their original cavity. Bulk flow (red) is directed opposite to the edge current ${ }^{15,16}$ (Supplementary Movie 3). d, Graph of the Union Jack double-lattice model in an antiferromagnetic state with zero net pillar circulation. Solid and dashed lines depict vortex-vortex and vortex-pillar interactions of respective strengths $J_{v}$ and $J_{\mathrm{p}}$. Vortices and pillars are colour-coded according to their spin. e, For supercritical gap widths $w>w_{\text {crit, }}$ extended domains of ferromagnetic order predominate (Supplementary Movie 2; $w=11 \mu \mathrm{m}$ ). Scale bar, $50 \mu \mathrm{m}$. f, PIV field within a ferromagnetic domain (Supplementary Movie 2). Largest arrows: $36 \mu \mathrm{m} \mathrm{s}^{-1}$. Scale bar, 20 $\mu \mathrm{m}$. $\mathbf{g}$, For $w>w_{\text {crit, }}$ bacteria forming the edge current (blue arrows) swim along the PDMS boundary through the gap, driving bulk flows (red) in the opposite directions, thereby aligning neighbouring vortex spins. h, Ferromagnetic state of the Union Jack lattice induced by edge current loops around the pillars. i, Trajectories of neighbouring spins ( $*$-symbols in $\mathbf{a}, \mathbf{e}$ ) fluctuate over time, signalling exploration of a fluctuating steady state under a non-zero effective temperature (top, antiferromagnetic; bottom, ferromagnetic). j, The zero of the spin-spin correlation $\chi$ at $w_{\text {crit }} \approx 8 \mu \mathrm{m}$ marks the phase transition. The best-fit Union Jack model (solid line) is consistent with the experimental data. $\mathbf{k}$, R.m.s. vortex spin $\left\langle V_{i}^{2}\right\rangle^{1 / 2}$ decreases with the gap size $w$, showing weakening of the circulation. R.m.s. pillar spin $\left\langle P_{j}^{2}\right\rangle^{1 / 2}$ increases with $w$, reflecting enhanced bacterial circulation around pillars. Each point in $\mathbf{j}, \mathbf{k}$ represents an average over $\geq 5$ movies in $3 \mu \mathrm{m}$ bins at $1.5 \mu \mathrm{m}$ intervals; vertical bars indicate standard errors (Methods).

on the vortex spins (Supplementary Fig. 1 and Supplementary Section 1), we conclude that the observed phase behaviour is caused by spin-spin interactions. However, although both phases possess a well-defined average vortex-vortex correlation, the individual spins fluctuate randomly over time as ordered domains split, merge and flip (Fig. 1i and Supplementary Figs 1 and 3) while the system explores configuration space inside a statistical steady state (Supplementary Sections 1 and 3). Thus, although the bacterial vortex spins $\left\{V_{i}(t)\right\}$ define a real-valued lattice field, the phenomenology of these continuous bacterial spin lattices is qualitatively similar to that of the classical 2D Ising model ${ }^{4}$ with discrete binary spin variables $s_{i} \in\{ \pm 1\}$, whose configurational probability at finite temperature $T=\left(k_{\mathrm{B}} \beta\right)^{-1}$ is described by a thermal Boltzmann distribution $\propto \exp \left(-\beta J \Sigma_{i \sim j} s_{i} s_{j}\right)$, where $J>0$ corresponds to ferromagnetic and $J<0$ to antiferromagnetic order. The detailed theoretical analysis below shows that the observed phases in the bacterial spin system can be understood quantitatively in terms of a generic quartic LFT comprising two dual interacting lattices. The introduction of a double lattice is necessitated by the microscopic structure of the underlying bacterial flows. By analogy with a lattice of interlocking cogs, one might have intuitively expected that the antiferromagnetic phase would generally be favoured, because only in this configuration does the bacterial flow along the cavity boundaries conform across the inter-cavity gap, avoiding the potentially destabilizing head-to-head collisions that would occur with opposing flows (Fig. 1b,c). However, the extent of the observed ferromagnetic phase highlights a competing biofluid-mechanical effect.

Just as the quantum Hall effect ${ }^{17}$ and the transport properties of graphene ${ }^{18,19}$ arise from electric edge currents, the opposing order regimes observed here are explained by the existence of analogous bacterial edge currents. At the boundary of an isolated flattened droplet of a bacterial suspension, a single layer of cellsan edge current-can be observed swimming against the bulk circulation $^{15,16}$. This narrow cell layer is key to the suspension dynamics: the hydrodynamics of the edge current circulating 
in one direction advects nearby cells in the opposite direction, which in turn dictate the bulk circulation by flow continuity through steric and hydrodynamic interactions ${ }^{16,27}$. Identical edge currents are present in our lattices (Supplementary Movie 3) and explain both order regimes as follows. In the antiferromagnetic regime, when $w<w_{\text {crit }}$, the bacterial edge current driving a particular vortex will pass over the gap without leaving the cavity (Fig. 1c). Interaction with a neighbouring edge current through the gap favours parallel flow, inducing counter-circulation of neighbouring vortices and therefore driving antiferromagnetic order (Fig. 1d). However, when $w>w_{\text {crit }}$, the edge currents can no longer pass over the gaps and instead wind around the star-shaped pillars dividing the cavities (Fig. 1g). A clockwise (resp. anticlockwise) bacterial edge current about a pillar induces anticlockwise (resp. clockwise) fluid circulation about the pillar in a thin region near its boundary. Flow continuity then induces clockwise (resp. anticlockwise) flow in all cavities adjacent to the pillar, resulting in ferromagnetic order (Fig. 1h). Thus by viewing the system as an anti-cooperative Union Jack lattice ${ }^{28,29}$ of both bulk vortex spins $V_{i}$ and near-pillar circulations $P_{j}$, we accommodate both order regimes: antiferromagnetism as indefinite circulations $P_{j}=0$ and alternating spins $V_{i}= \pm V$ (Fig. 1d), and ferromagnetism as definite circulations $P_{j}=-P<0$ and uniform spins $V_{i}=V>0$ (Fig. 1h). To verify these considerations, we determined the net near-pillar circulation $P_{j}(t)$ using PIV (Methods) and found that the r.m.s. circulation $\left\langle P_{j}(t)^{2}\right\rangle^{1 / 2}$ shows the expected monotonic increase as the inter-cavity gap widens (Fig. 1k).

Competition between the vortex-vortex and vortex-pillar interactions determines the resultant order regime. Their relative strengths can be inferred by mapping each experiment onto a continuous-spin Union Jack lattice (Fig. 1d,h). In this model, the interaction energy of the time-dependent vortex spins $\mathbf{V}=\left\{V_{i}\right\}$ and pillar circulations $\mathbf{P}=\left\{P_{j}\right\}$ is defined by the LFT Hamiltonian

$$
\begin{aligned}
H(\mathbf{V}, \mathbf{P})= & -J_{\mathrm{v}} \sum_{V_{i} \sim V_{j}} V_{i} V_{j}-J_{\mathrm{P}} \sum_{V_{i} \sim P_{j}} V_{i} P_{j} \\
& +\sum_{V_{i}}\left(\frac{1}{2} a_{v} V_{i}^{2}+\frac{1}{4} b_{v} V_{i}^{4}\right)+\sum_{P_{j}} \frac{1}{2} a_{\mathrm{p}} P_{j}^{2}
\end{aligned}
$$

The first two sums are vortex-vortex and vortex-pillar interactions with strengths $J_{\mathrm{v}}, J_{\mathrm{p}}<0$, where $\sim$ denotes adjacent lattice pairs. The last two sums are individual vortex and pillar circulation potentials. Vortices must be subject to a quartic potential function with $b_{v}>0$ to allow for a potentially double-welled potential if $a_{v}<0$, encoding the observed symmetry breaking into spontaneous circulation in the absence of other interactions ${ }^{15,27}$. In contrast, our data analysis implies that pillar circulations are sufficiently described by a quadratic potential of strength $a_{\mathrm{p}}>0$ (Supplementary Fig. 4 and Supplementary Section 4). To account for the experimentally observed spin fluctuations (Fig. 1i and Supplementary Fig. 1), we model the dynamics of the lattice fields $\mathbf{V}$ and $\mathbf{P}$ through the coupled stochastic differential equations (SDEs)

$$
\begin{aligned}
& \mathrm{d} \mathbf{V}=-(\partial H / \partial \mathbf{V}) \mathrm{d} t+\sqrt{2 T_{\mathrm{v}}} \mathrm{d} \mathbf{W}_{\mathrm{v}} \\
& \mathrm{d} \mathbf{P}=-(\partial H / \partial \mathbf{P}) \mathrm{d} t+\sqrt{2 T_{\mathrm{p}}} \mathrm{d} \mathbf{W}_{\mathrm{p}}
\end{aligned}
$$

where $\mathbf{W}_{\mathrm{v}}$ and $\mathbf{W}_{\mathrm{p}}$ are vectors of uncorrelated Wiener processes representing intrinsic and thermal fluctuations. The overdamped dynamics in equations (2) and (3) neglects dissipative Onsagertype cross-couplings, as the dominant contribution to friction stems from the nearby no-slip PDMS boundaries (Supplementary Section 7). The parameters $T_{\mathrm{v}}$ and $T_{\mathrm{p}}$ set the strength of random perturbations from energy-minimizing behaviour. In the equilibrium limit when $T_{\mathrm{v}}=T_{\mathrm{p}}=T$, the stationary statistics of the solutions of equations (2) and (3) obey the Boltzmann distribution $\propto \mathrm{e}^{-H / T}$. We inferred all seven parameters of the full SDE model for each experiment by linear regression on a discretization of the SDEs (Supplementary Fig. 2 and Supplementary Section 2). The differing sublattice temperatures $T_{\mathrm{v}} \neq T_{\mathrm{p}}$ found show that the system is not in thermodynamic equilibrium owing to its active microscopic constituents (Supplementary Fig. 2). Instead, the system is in a pseudo-equilibrium statistical steady state (Supplementary Section 1), which we will soon show can be reduced to an equilibrium-like description. As a cross-validation, we fitted appropriate functions of gap width $w$ to these estimates and simulated the resulting SDE model over a range of $w$ on a $6 \times 6$ lattice concordant with the observations (Supplementary Section 3). The agreement between experimental data and the numerically obtained vortex-vortex correlation $\chi(w)$ supports the validity of the doublelattice model and its underlying approximations (Fig. 1j).

To reconnect with the classical 2D Ising model and understand better the experimentally observed phase transition, we project the Hamiltonian (1) onto an effective square lattice model by making a mean-field assumption for the pillar circulations. In the experiments, $P_{i}$ is linearly correlated with the average spin of its vortex neighbours $\left[P_{i}\right]_{V}=(1 / 4) \Sigma_{j: V_{j} \sim P_{i}} V_{j}$, with a constant of proportionality $-\alpha<0$ only weakly dependent on gap width (Supplementary Fig. 4 and Supplementary Section 4). Replacing effectively $P_{i} \rightarrow-\alpha\left[P_{i}\right]_{V}$ as a mean-field variable in the model eliminates all pillar circulations, yielding a standard quartic LFT for V (see Supplementary Section 4 for a detailed derivation). The mean-field dynamics are then governed by the reduced SDE $\mathrm{d} \mathbf{V}=-(\partial \hat{H} / \partial \mathbf{V}) \mathrm{d} t+\sqrt{2 T} \mathrm{~d} \mathbf{W}$ with effective temperature $T \approx T_{\mathrm{v}}+4 T_{\mathrm{p}} J_{\mathrm{p}}^{2} / a_{\mathrm{p}}^{2}$ and energy

$$
\hat{H}(\mathbf{V})=-J \sum_{V_{i} \sim V_{j}} V_{i} V_{j}+\sum_{V_{i}}\left(\frac{1}{2} a V_{i}^{2}+\frac{1}{4} b V_{i}^{4}\right)
$$

which has steady-state probability density $p(\mathbf{V}) \propto \mathrm{e}^{-\beta \hat{H}}$ with $\beta=1 / T$, and where $a=a_{v}-4 J_{\mathrm{p}}^{2} / a_{\mathrm{p}}$ and $b=b_{v}$. Note that in the limit $a \rightarrow-\infty$ and $b \rightarrow+\infty$ with $a / b$ fixed, the classical two-state Ising model is recovered by identifying $s_{i}=V_{i} / \sqrt{|a| / b} \in\{ \pm 1\}$. The reduced coupling constant $J$ relates to those of the double-lattice model $\left(J_{\mathrm{v}}, J_{\mathrm{p}}\right)$ in the thermodynamic limit as $J \approx J_{\mathrm{v}}-(1 / 2) \alpha J_{\mathrm{p}}$ (Supplementary Section 4$)$, making manifest how competition between $J_{\mathrm{v}}$ and $J_{\mathrm{p}}$ can result in both antiferromagnetic $\left(\left|J_{\mathrm{v}}\right|>(1 / 2) \alpha\left|J_{\mathrm{p}}\right|\right)$ or ferromagnetic $\left(\left|J_{\mathrm{v}}\right|<(1 / 2) \alpha\left|J_{\mathrm{p}}\right|\right)$ behaviour. We estimated $\beta J, \beta a$ and $\beta b$ for each experiment by directly fitting the effective one-spin potential $\mathcal{V}^{\text {eff }}\left(V \mid[V]_{V}\right)=-4 \beta J V[V]_{V}+(1 / 2) \beta a V^{2}+(1 / 4) \beta b V^{4}$ via the $\log$-likelihood $\log p\left(V \mid[V]_{V}\right)=-\mathcal{V}^{\text {eff }}+$ const (Fig. 2, Supplementary Fig. 5 and Supplementary Section 5). These estimates match those obtained independently using SDE regression methods (Fig. 2a-c and Supplementary Section 5), and show the transition from antiferromagnetic interaction $(\beta J<0)$ to ferromagnetic interaction $(\beta J>0)$ at $w_{\text {crit }}$ (Fig. 2a). As the gap width increases, the energy barrier to spin change falls (Fig. 2b) and the magnitude of the lowest energy spin decreases (Fig. 2c) as a result of weakening confinement within each cavity, visible as a flattening of the one-spin effective potential $\mathcal{V}^{\text {eff }}$ (Fig. $2 d-f$ and Supplementary Fig. 5).

Experiments on lattices of different symmetry groups lend further insight into the competition between edge currents and bulk flow. Unlike their square counterparts, triangular lattices cannot support antiferromagnetic states without frustration. Therefore, ferromagnetic order should be enhanced in a triangular bacterial spin lattice. This is indeed observed in our experiments: at moderate gap size $w \lesssim 18 \mu \mathrm{m}$, we found exclusively a highly 


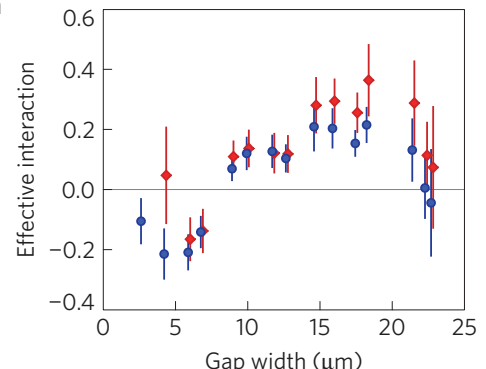

d

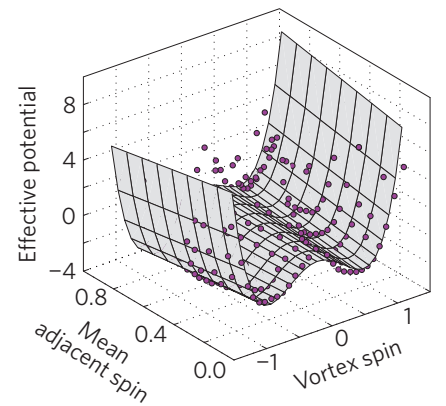

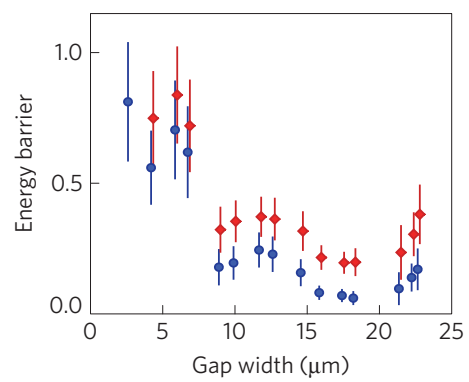

e

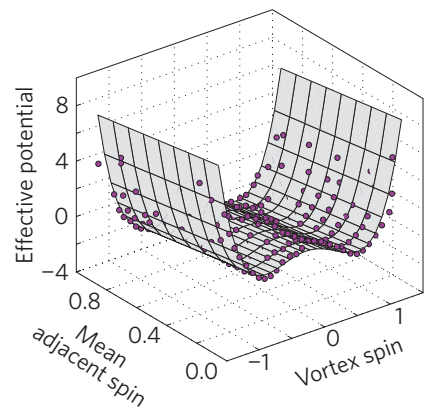

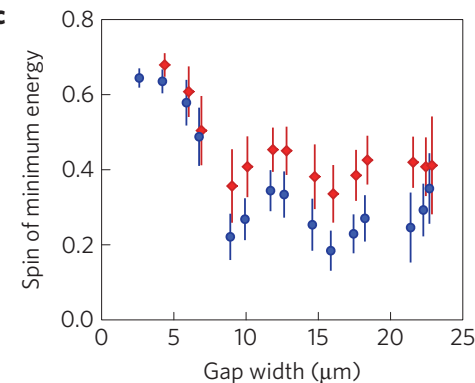

f

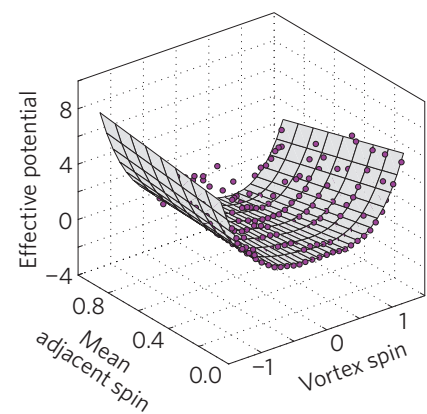

Figure 2 | Best-fit mean-field LFT model captures the phase transition in the square lattice. $\mathbf{a}$, A sign change of the effective interaction $\beta J$ signals the transition from antiferro- to ferromagnetic states. b. The effective energy barrier, $\beta a^{2} /(4 b)$ when $a<0$ and zero when $a>0$ (Supplementary Section 5 ), decreases with the gap size $w$, reflecting increased susceptibility to fluctuations. c, The spin $V_{\text {min }}$ minimizing the single-spin potential (Supplementary Section 5) decreases with $w$ in agreement with the decrease in the r.m.s. vortex spin (Fig. 1k). Each point in a-c represents an average over $\geq 5$ movies in $3 \mu \mathrm{m}$ bins at $1.5 \mu \mathrm{m}$ intervals; blue circles are from distribution fitting, red diamonds are from SDE regression, and vertical bars indicate standard errors (Methods). d-f, Examples of the effective single-spin potential $\mathcal{V}^{\text {eff }}$ conditional on the mean spin of adjacent vortices [V]V. Data (points) and estimated potential (surface) for three movies with gap widths 6,10 and $17 \mu \mathrm{m}$.
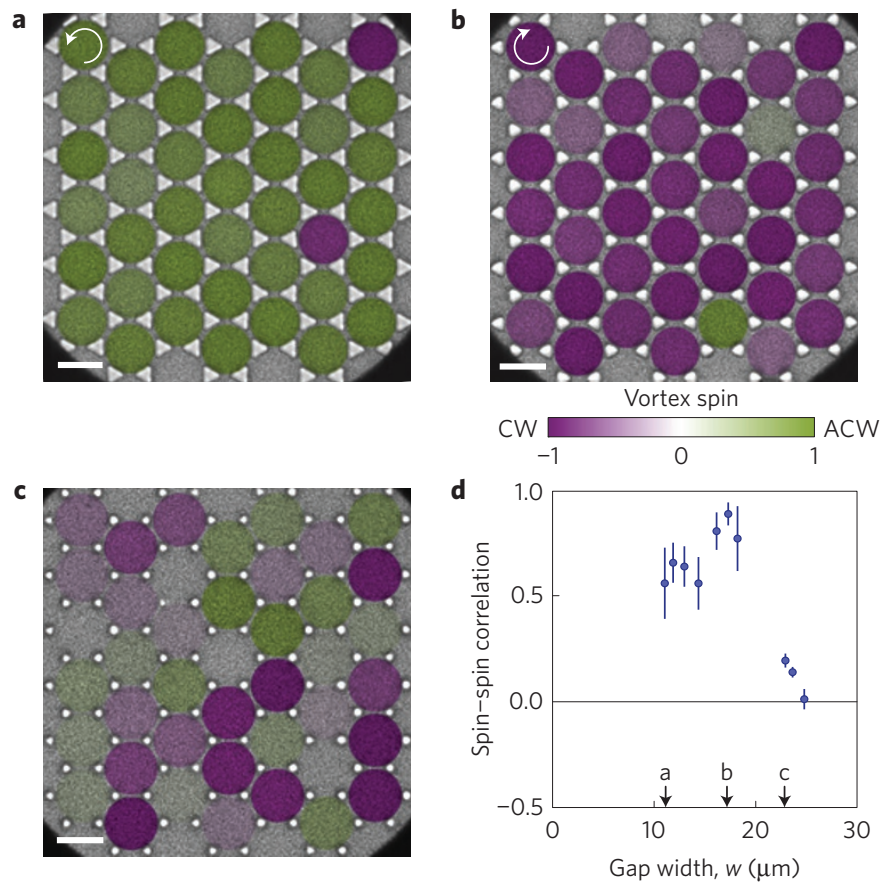

Figure 3 | Frustration in triangular lattices determines the preferred order. $\mathbf{a}, \mathbf{b}$, Triangular lattices favour ferromagnetic states of either handedness (Supplementary Movie 4). Vortices are colour-coded by spin. c, At the largest gap size, bacterial circulation becomes unstable. Scale bar, $50 \mu \mathrm{m}$. d, The spin-spin correlation $\chi$ shows strongly enhanced ferromagnetic order compared with the square lattice (Fig. 1j). Each point represents an average over $\geq 5$ movies in $3 \mu \mathrm{m}$ bins at $1.5 \mu \mathrm{m}$ intervals; vertical bars indicate standard errors (Methods). robust ferromagnetic phase of either handedness (Fig. 3a,b,d and Supplementary Movie 4), reminiscent of quantum vortex lattices in Bose-Einstein condensates ${ }^{30}$. At comparable gap size, the spin correlation is approximately four to eight times larger than in the square lattice. Increasing the gap size beyond $20 \mu \mathrm{m}$ eventually destroys the spontaneous circulation within the cavities and a disordered state prevails (Fig. 3c,d), with a sharper transition than for the square lattices (Fig. 1j). Conversely, a 1D line lattice exclusively exhibits antiferromagnetic order as the suspension is unable to maintain the very long uniform edge currents that would be necessary to sustain a ferromagnetic state (Supplementary Fig. 6 and Supplementary Section 6). These results manifest the importance of lattice geometry and dimensionality for vortex ordering in bacterial spin lattices, in close analogy with their electromagnetic counterparts.

Understanding the ordering principles of microbial matter is a key challenge in active materials design ${ }^{13}$, quantitative biology and biomedical research. Improved prevention strategies for pathogenic biofilm formation, for example, will require detailed knowledge of how bacterial flows interact with complex porous surface structures to create the stagnation points at which biofilms can nucleate. Our study shows that collective excitations in geometrically confined bacterial suspensions can spontaneously organize in phases of magnetic order that can be robustly controlled by edge currents. These results demonstrate fundamental similarities with a broad class of widely studied quantum systems $\mathrm{s}^{17,19,30}$, suggesting that theoretical concepts originally developed to describe magnetism in disordered media could potentially capture microbial behaviours in complex environments. Future studies may try to explore further the range and limits of this promising analogy.

\section{Methods}

Methods and any associated references are available in the online version of the paper. 
Received 11 June 2015; accepted 13 November 2015; published online 4 January 2016

\section{References}

1. Schrödinger, E. What is Life? (Cambridge Univ. Press, 1944).

2. Vicsek, T. \& Zafeiris, A. Collective motion. Phys. Rep. 517, 71-140 (2012).

3. Marchetti, M. C. et al. Hydrodynamics of soft active matter. Rev. Mod. Phys. 85, 1143-1189 (2013).

4. Kardar, M. Statistical Physics of Fields (Cambridge Univ. Press, 2007).

5. Mermin, N. D. The topological theory of defects in ordered media. Rev. Mod. Phys. 51, 591-648 (1979).

6. Ben Jacob, E., Becker, I., Shapira, Y. \& Levine, H. Bacterial linguistic communication and social intelligence. Trends Microbiol. 12, 366-372 (2004).

7. Volfson, D., Cookson, S., Hasty, J. \& Tsimring, L. S. Biomechanical ordering of dense cell populations. Proc. Natl Acad. Sci. USA 150, 15346-15351 (2008).

8. Riedel, I. H., Kruse, K. \& Howard, J. A self-organized vortex array of hydrodynamically entrained sperm cells. Science 309, 300-303 (2005).

9. Dunkel, J. et al. Fluid dynamics of bacterial turbulence. Phys. Rev. Lett. 110, 228102 (2013).

10. Wu, J., Roman, A.-C., Carvajal-Gonzalez, J. M. \& Mlodzik, M. Wg and Wnt4 provide long-range directional input to planar cell polarity orientation in drosophila. Nature Cell Biol. 15, 1045-1055 (2013).

11. Katz, Y., Ioannou, C. C., Tunstro, K., Huepe, C. \& Couzin, I. D. Inferring the structure and dynamics of interactions in schooling fish. Proc. Natl Acad. Sci. USA 108, 18720-18725 (2011).

12. Cavagna, A. et al. Scale-free correlations in starling flocks. Proc. Natl Acad. Sci. USA 107, 11865-11870 (2010).

13. Sanchez, T., Chen, D. T. N., DeCamp, S. J., Heymann, M. \& Dogic, Z. Spontaneous motion in hierarchically assembled active matter. Nature 491, 431-434 (2012).

14. Sokolov, A. \& Aranson, I. S. Physical properties of collective motion in suspensions of bacteria. Phys. Rev. Lett. 109, 248109 (2012).

15. Wioland, H., Woodhouse, F. G., Dunkel, J., Kessler, J. O. \& Goldstein, R. E. Confinement stabilizes a bacterial suspension into a spiral vortex. Phys. Rev. Lett. 110, 268102 (2013).

16. Lushi, E., Wioland, H. \& Goldstein, R. E. Fluid flows created by swimming bacteria drive self-organization in confined suspensions. Proc. Natl Acad. Sci. USA 111, 9733-9738 (2014).

17. Büttiker, M. Absence of backscattering in the quantum Hall effect in multiprobe conductors. Phys. Rev. B 38, 9375-9389 (1988).

18. Kane, C. L. \& Mele, E. J. Quantum spin Hall effect in graphene. Phys. Rev. Lett. 95, 226801 (2005).

19. Castro Neto, A. H., Guinea, F., Peres, N. M. R., Novoselov, K. S. \& Geim, A. K. The electronic properties of graphene. Rev. Mod. Phys. 81, 109-162 (2009).
20. Wilson, K. G. Confinement of quarks. Phys. Rev. D 10, 2445-2459 (1974).

21. Glendenning, N. K. Compact Stars: Nuclear Physics, Particle Physics, and General Relativity (Springer, 2000).

22. Battye, R. A. \& Sutcliffe, P. M. Skyrmions with massive pions. Phys. Rev. C 73, 055205 (2006).

23. Nagaosa, N. \& Tokura, Y. Topological properties and dynamics of magnetic skyrmions. Nature Nanotech. 8, 899-911 (2013).

24. Novoselov, K. S. et al. Two-dimensional gas of massless Dirac fermions in graphene. Nature 438, 197-200 (2005).

25. Drut, J. E. \& Lähde, T. A. Lattice field theory simulations of graphene. Phys. Rev. B 79, 165425 (2009).

26. Fernández, R., Fröhlich, J. \& Sokal, A. D. Random Walks, Critical Phenomena, and Triviality in Quantum Field Theory (Springer, 1992).

27. Woodhouse, F. G. \& Goldstein, R. E. Spontaneous circulation of confined active suspensions. Phys. Rev. Lett. 109, 168105 (2012).

28. Vaks, V. G., Larkin, A. I. \& Ovchinnikov, Y. N. Ising model with interaction between non-nearest neighbors. Sov. Phys. JETP 22, 820-826 (1966).

29. Stephenson, J. Ising model with antiferromagnetic next-nearest-neighbor coupling: spin correlations and disorder points. Phys. Rev. B 1, 4405-4409 (1970).

30. Abo-Shaeer, J. R., Raman, C., Vogels, J. M. \& Ketterle, W. Observation of vortex lattices in Bose-Einstein condensates. Science 292, 476-479 (2001).

\section{Acknowledgements}

We thank V. Kantsler and E. Lushi for assistance and discussions. This work was supported by European Research Council Advanced Investigator Grant 247333 (H.W. and R.E.G.), EPSRC (H.W. and R.E.G.), an MIT Solomon Buchsbaum Fund Award (J.D.) and an Alfred P. Sloan Research Fellowship (J.D.).

\section{Author contributions}

All authors designed the research and collaborated on theory. H.W. performed experiments and PIV analysis. H.W. and F.G.W. analysed PIV data and performed parameter inference. F.G.W. and J.D. wrote simulation code. All authors wrote the paper.

\section{Additional information}

Supplementary information is available in the online version of the paper. Reprints and permissions information is available online at www.nature.com/reprints. Correspondence and requests for materials should be addressed to R.E.G.

\section{Competing financial interests}

The authors declare no competing financial interests. 


\section{Methods}

Experiments. Wild-type Bacillus subtilis cells (strain 168) were grown in Terrific Broth (Sigma). A monoclonal colony was transferred from an agar plate to $25 \mathrm{ml}$ of medium and left to grow overnight at $35^{\circ} \mathrm{C}$ on a shaker. The culture was diluted 200 -fold into fresh medium and harvested after approximately $5 \mathrm{~h}$, when more than $90 \%$ of the bacteria were swimming, as visually verified on a microscope. $10 \mathrm{ml}$ of the suspension was then concentrated by centrifugation at $1,500 \mathrm{~g}$ for $10 \mathrm{~min}$, resulting in a pellet with volume fraction approximately $20 \%$ which was used without further dilution.

The microchambers were made of polydimethyl siloxane (PDMS) bound to a glass coverslip by oxygen plasma etching. These comprised a square, triangular or linear lattice of $\sim 18$ - $\mu \mathrm{m}$-deep circular cavities with $60 \mu \mathrm{m}$ between centres, each of diameter $\sim 50 \mu \mathrm{m}$, connected by $4-25-\mu \mathrm{m}$-wide gaps for linear and square lattices (Fig. 1a,e and Supplementary Fig. 6) and 10-25- $\mu \mathrm{m}$-wide gaps for triangular lattices (Fig. $3 \mathrm{a}-\mathrm{c}$ ). The smallest possible gap size was limited by the fidelity of the etching. Approximately $5 \mathrm{ul}$ of the concentrated suspension was manually injected into the chamber using a syringe. Both inlets were then sealed to prevent external flow. We imaged the suspension on an inverted microscope (Zeiss, Axio Observer Z1) under bright-field illumination, through a $40 \times$ oil-immersion objective. Movies $10 \mathrm{~s}$ in length were recorded at 60 f.p.s. on a high-speed camera (Photron Fastcam SA3) at 4 and $8 \mathrm{~min}$ after injection. Although the PDMS lattices were typically $\sim 15$ cavities across, to avoid boundary effects and to attain the pixel density necessary for PIV we imaged a central subregion spanning $6 \times 6$ cavities for square lattices, $7 \times 6$ cavities for triangular lattices, and 7 cavities for linear lattices (multiple of which were captured on a single slide).

Fluorescence in Supplementary Movie 3 was achieved by labelling the membranes of a cell subpopulation with fluorophore FM4-64 following the protocol of Lushi et al. ${ }^{16}$ The suspension was injected into an identical triangular lattice as in the primary experiments and imaged at 5.6 f.p.s. on a spinning-disc confocal microscope through a $63 \times$ oil-immersion objective.

Analysis. For each frame of each movie, the bacterial suspension flow field $\mathbf{u}(x, y, t)$ was measured by standard particle image velocimetry (PIV) without time averaging, using a customized version of mPIV (http://www.oceanwave.jp/ softwares/mpiv). PIV subwindows were $16 \times 16$ pixels with $50 \%$ overlap, yielding $\sim 150$ vectors per cavity per frame. Cavity regions were identified in each movie by manually placing the centre and radius of the bottom left cavity, measuring vectors to its immediate neighbours, and repeatedly translating to generate the full grid. Pillar edges were then calculated from the cavity grid and the gap width (measured as the minimum distance between adjacent pillars).
The spin $V_{i}(t)$ of each cavity $i$ at time $t$ is defined as the normalized planar angular momentum

$$
V_{i}(t)=\frac{\hat{\mathbf{z}} \cdot\left[\sum_{(x, y)_{i}} \mathbf{r}_{i}(x, y) \times \mathbf{u}(x, y, t)\right]}{\bar{U} \sum_{(x, y)_{i}}\left|\mathbf{r}_{i}(x, y)\right|}
$$

where $\mathbf{r}_{i}(x, y)$ is the vector from the cavity centre to $(x, y)$, and sums run over all PIV grid points $(x, y)_{i}$ inside cavity $i$. For each movie, we normalize velocities by the root-mean-square (r.m.s.) suspension velocity $\bar{U}=\left\langle\mathbf{u}(x, y, t)^{2}\right\rangle^{1 / 2}$, where the average is over all grid points $(x, y)$ and all times $t$, to account for the effects of variable oxygenation on motility ${ }^{9}$; we found an ensemble average $\mathbb{E}[\bar{U}]=12.1 \mu \mathrm{m} \mathrm{s}^{-1}$ with s.d. $3.6 \mu \mathrm{m} \mathrm{s}^{-1}$ over all experiments. This definition has $V_{i}(t)>0$ for anticlockwise spin and $V_{i}(t)<0$ for clockwise spin. A vortex of radially independent speed-that is, $\mathbf{u}(x, y, t)=u \hat{\boldsymbol{\theta}}$, where $\hat{\boldsymbol{\theta}}$ is the azimuthal unit vector-has $V_{i}(t)= \pm 1$; conversely, randomly oriented flow has $V_{i}(t)=0$. The average spin-spin correlation $\chi$ of a movie is then defined as

$$
\chi=\left\langle\frac{\sum_{i \sim j} V_{i}(t) V_{j}(t)}{\sum_{i \sim j}\left|V_{i}(t) V_{j}(t)\right|}\right\rangle
$$

where $\Sigma_{i \sim j}$ denotes a sum over pairs $\{i, j\}$ of adjacent cavities and $\langle\cdot\rangle$ denotes an average over all frames. If all vortices share the same sign, then $\chi=1$

(ferromagnetism); if each vortex is of opposite sign to its neighbours, then $\chi=-1$ (antiferromagnetism); if the vortices are uniformly random, then $\chi=0$. Similarly, the circulation $P_{j}(t)$ about pillar $j$ at time $t$ is defined as the normalized average tangential velocity

$$
P_{j}(t)=\frac{\sum_{(x, y) j} \mathbf{u}(x, y, t) \cdot \hat{\mathbf{t}}_{j}(x, y)}{\bar{U} \sum_{(x, y)_{j}} 1}
$$

where $\hat{\mathbf{t}}_{j}(x, y)$ is the unit vector tangential to the pillar, and sums run over PIV grid points $(x, y)_{j}$ closer than $5 \mu \mathrm{m}$ to the pillar $j$.

Results presented are typically averaged in bins of fixed gap width. All plots with error bars use $3 \mu \mathrm{m}$ bins, calculated every $1.5 \mu \mathrm{m}$ ( $50 \%$ overlap), and bins with fewer than five movies were excluded. Error bars denote standard error. Bin counts for square lattices (Figs $1 \mathrm{j}, \mathrm{k}$ and $2 \mathrm{a}-\mathrm{c}$ and Supplementary Figs 4 and 7) are 8, 8, 13, $14,21,27,27,22,18,22,20,11,7,13$, 7; bin counts for triangular lattices (Fig. 3d) are $5,14,16,13,16,15,5,5,10,7$; and bin counts for linear lattices (Supplementary Fig. 6) are 5, 7, 8, 8, 9, 9, 6, 5, 6, 7, 6, 8, 9, 5, 6, 5, 6 . 